\title{
Greenhouse Monitoring System Using IOT
}

\author{
D.O.I-10.51201/12371 \\ https://doi.org/10.51201/12371
}

\author{
Sahil Kumar ${ }^{1}$, Raj Ranjan Kumar², Sonu Kumar ${ }^{3}$, Rohit Kothawale ${ }^{4}$, \\ Prof. Y. D. Chincholkar ${ }^{5}$ \\ Student ,Electronics \& Telecommunication, Sinhgad College of \\ Engineering,Pune,India ${ }^{1,2,3,4}$
}

Guide, Electronics \& Telecommunication, Sinhgad College of Engineering,

Pune, India ${ }^{5}$

\begin{abstract}
This project is based on human less maintenance of agriculture . In this the moisture of the soil, temperature and light intensity of the place will maintained automatically. Although we can predict the upcoming weather, there can be changes like sudden rain or harsh sunlight. These unpredicted changes in weather can destroy the crops. To avoid this, this system will automatically maintain the conditions which are favorable for the crops. If there is sudden rain and if the temperature is too low for the crops, the system will turn on the heat lamp due to which the temperature will be maintained. If due to high temperature, the moisture of the soils gets reduced, the system will turn on the water pump to maintain the moisture. Some crops need specific amount of light. If there is a situation that the surrounding light is low for the crops, then the system will turn on the light bulb to maintain the light intensity. In all these situations the farmer need not to run behind things like turning on water pump, light bulb, fan, etc. This system will take care of the maintenance of agriculture. All this data will be transmitted to the mobile device of the farmer.
\end{abstract}

Keywords: Microcontroller, Led, Soil Moisture Sensor, Temperature Sensor, Light Dependent Resistor, DC Motor, Servo Motor, etc.

\section{INTRODUCTION}

Sudden changes in weather are not favourable for agriculture. Unpredicted rain can destroy the crops. Or less rain can dry off the crops. High temperatures are not good for crops. Some crops even require specific amount of light. Less amount of light can affect the proper growth of the crops. To prevent all these things, the farmers need to turn on and off the water pump, light source, fan, etc. simultaneously. Such situations can be difficult for farmers. This system is developed to ease the work of farmers. The farmer need not run behind all these things. All the data of the sensors will be transmitted to the farmer's mobile. Due to this system, requirement of manpower is reduced. This system will help farmers for plantation on large scale with less number of labours. The soil moisture sensor is a sensor which varies the value when it contacts the moisture. basically it is a resistor that works on moisture condition. when the moisture is more then the value of resistance will decrease and when the moisture is less then the resistance value is more. Whenever we go out of town for few days, we always used to worry about our plants as they need water on regular basis. This system automatically provides water to your plants and keep you updated by sending message to your cell phone. This is a Completely Automated System and there is no need of manpower to control the system. Arduino is used for controlling the whole process and GSM module is used for sending alert messages to user on his Cell phone. 


\section{PROPOSED SYSTEM}

Below shown is the block diagram representation of the "Greenhouse Monitoring System Using IOT".

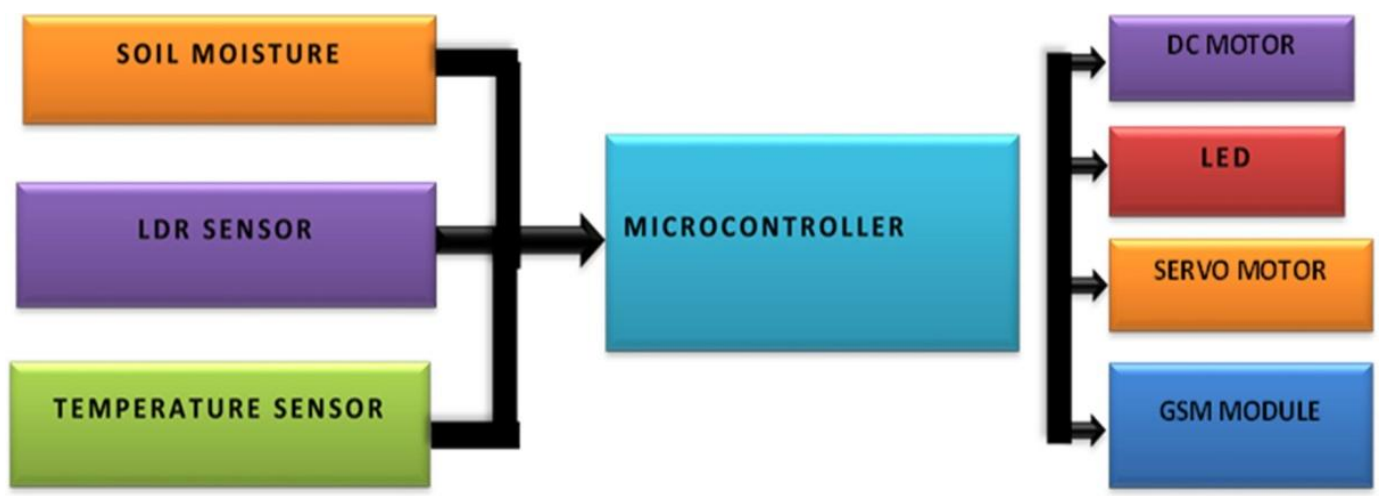

The system consists of total five blocks, the function of the five blocks are as follows:

1. LDR Sensor : A Light Dependent Resistor (LDR) is also called a photo resistor or a cadmium sulfide (CdS) cell. It is also called a photoconductor. It is basically a photocell that works on the principle of photoconductivity. The passive component is basically a resistor whose resistance value decreases when the intensity of light decreases. In this system the purpose of the sensor is to detect the light approaching from the vehicle in front and send the corresponding signal to the microcontroller. Below shown is a working diagram of LDR sensor for better understanding.
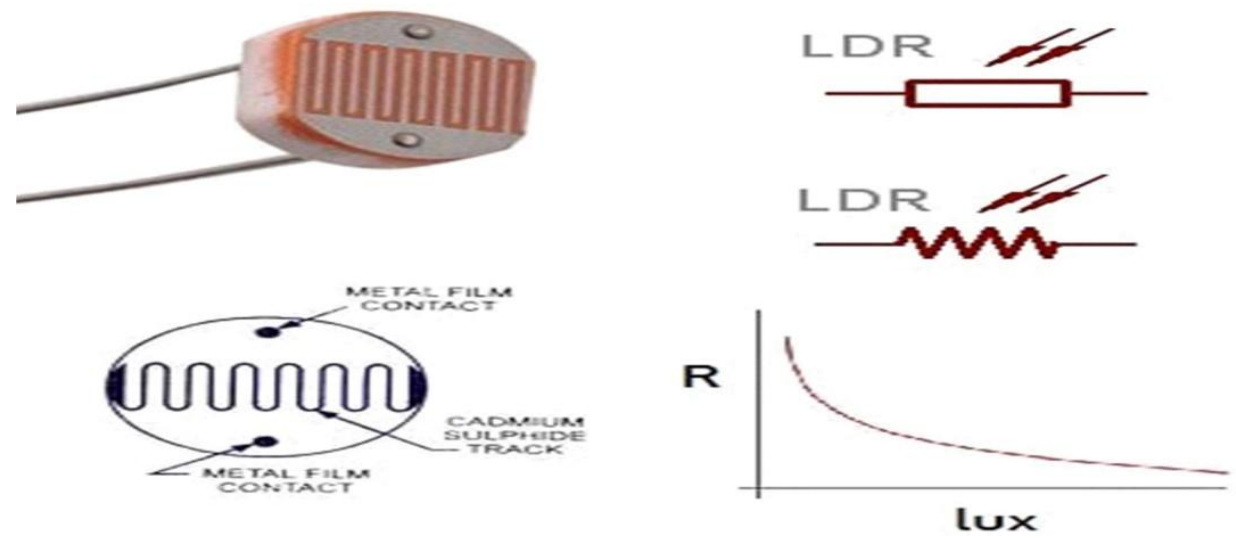

2. Microcontroller : A microcontroller is a compact integrated circuit designed to govern a specific operation in an embedded system. In this system we are using ARDUINO UNO as microcontroller. The purpose of choosing this as a microcontroller is due to its compact size and number of input/output pins available ( 14 digital and 6 analog pins available) which will make future development of the system of the easy and compact alongside. 


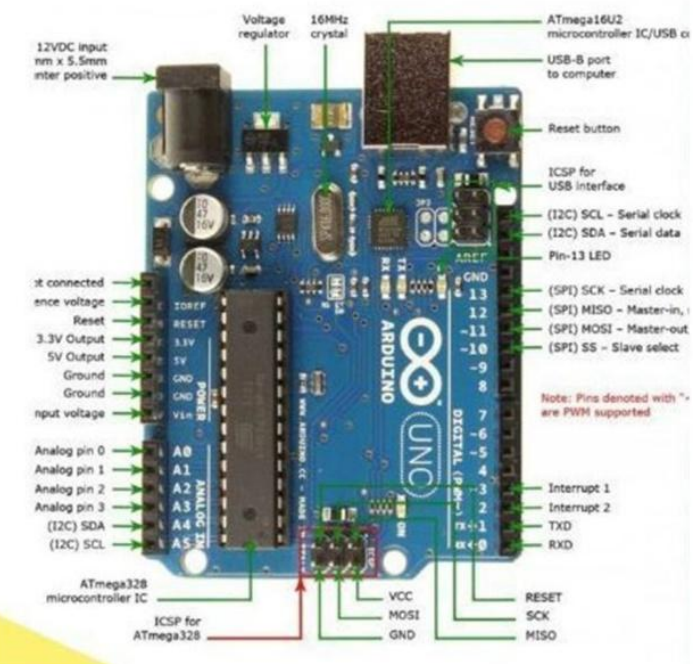

3. Soil Moisture Sensor: Soil moisture sensors measure the volumetric water content in soil. Since the direct gravimetric measurement of free soil moisture requires removing, drying, and weighing of a sample, soil moisture sensors measure the volumetric water content indirectly by using some other property of the soil, such as electrical resistance, dielectric constant, or interaction with neutrons, as a proxy for the moisture content.

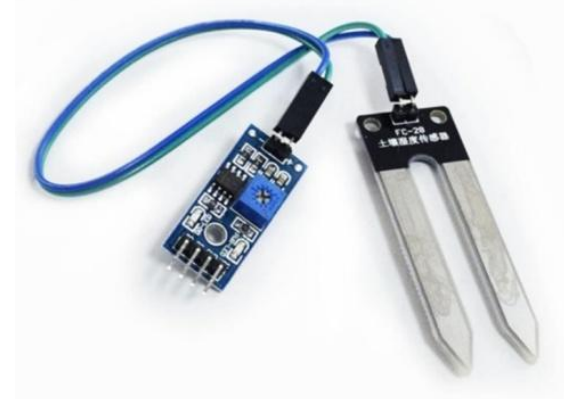

4. Servo Motor: Servo motor works on the PWM ( Pulse Width Modulation ) principle, which means its angle of rotation is controlled by the duration of pulse applied to its control PIN. Basically servo motor is made up of DC motor which is controlled by a variable resistor (potentiometer) and some gears.

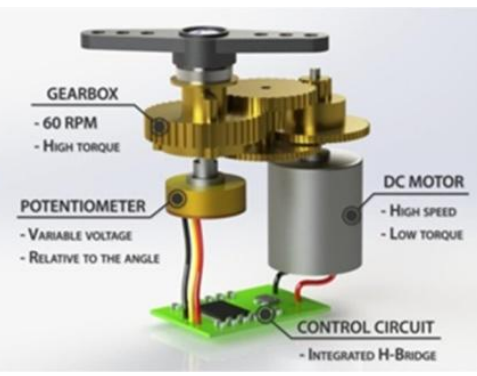


5. Temperature Sensor: LM35 is a precession Integrated circuit Temperature sensor, whose output voltage varies, based on the temperature around it. It is a small and cheap IC which can be used to measure temperature anywhere between $-55^{\circ} \mathrm{C}$ to $150^{\circ} \mathrm{C}$. It can easily be interfaced with any Microcontroller that has ADC function or any development platform like Arduino .

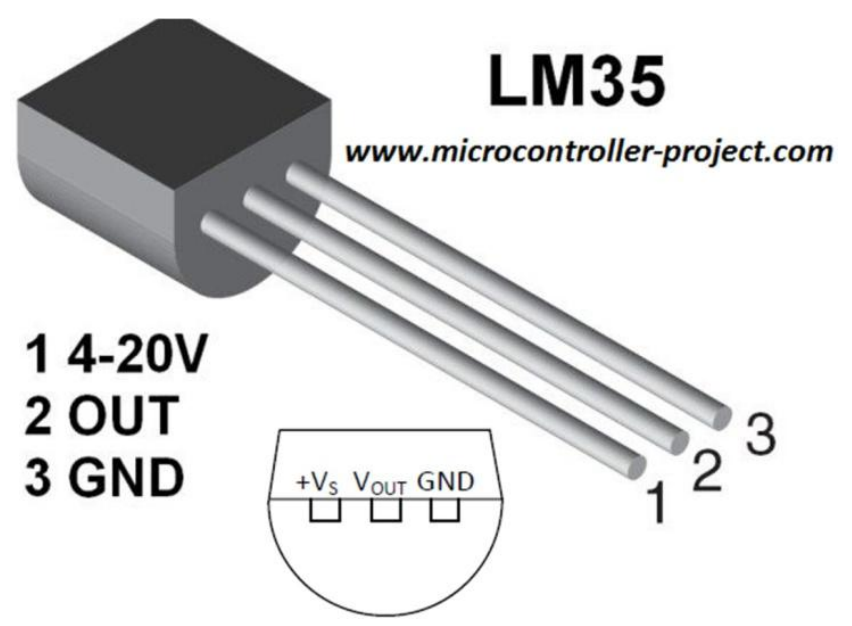


6. DC Motor : A DC motor is any of a class of rotary electrical motors that converts direct current electrical energy into mechanical energy. The most common types rely on the forces produced by magnetic fields. Nearly all types of DC motors have some internal mechanism, either electromechanical or electronic, to periodically change the direction of current in part of the motor.

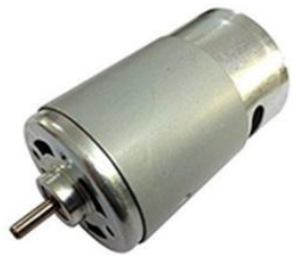

7. GSM Module: A GSM module or a GPRS module is a chip or circuit that will be used to establish communication between a mobile device or a computing machine and a GSM or GPRS system. GSM (Global System for Mobile Communications, originally Groupe Spécial Mobile), is a standard developed by the European Telecommunications Standards Institute (ETSI).

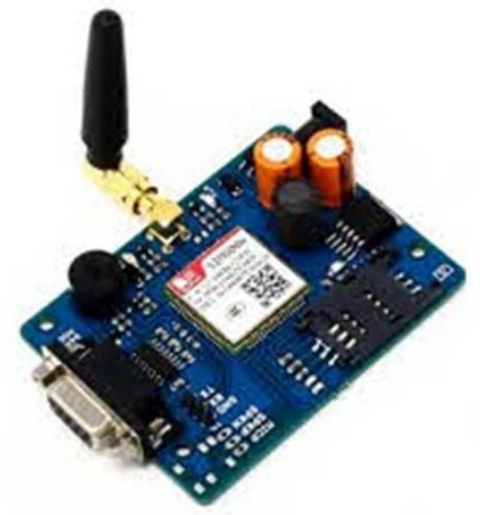

\section{NEED OF SYSTEM}

Temperature Sensor is used to detect the temperature inside the greenhouse. Reading from the sensor is sent to the microcontroller. The microcontroller is connected to different relays. One of the relays is connected to a blower. If the temperature is above or below the threshold value, the microcontroller would send signals to turn ON the Fan. Light Dependent Resistor Sensor is used to detect the amount of sunlight inside the greenhouse. Reading from the sensor is sent to the microcontroller. If the Sunlight is above the threshold value, the microcontroller would send signals to turn off the led bulb, in real-time, be a 'shade' that would reduce the amount of Sunlight. Similarly, the Humidity sensor is used to detect the humidity value and the Soil moisture sensor is used to detect the soil moisture. If the humidity value detected by the sensor is above the threshold value or if the soil moisture reduces, the microcontroller would turn open the water outlet to increase the moisture in the soil. For demo purposes, we have connected a DC motor in place of blower and water outlet. 


\section{WORKING}

1) Temperature Sensor: The temperature sensor readings will be continuously given as input to the microcontroller. When the reading is more than the threshold, the microcontroller will turn on the fan (DC Motor) and hence, the temperature will be maintained.

The basic principle of working of the temperature sensors is the voltage across the diode terminals. If the voltage increases, the temperature also rises, followed by a voltage drop between the transistor terminals of base and emitter in a diode.

Range :- 20 to 80 Degree Celsius. The temperature sensors are used for verifying design assumptions that will promote safer and economical design and construction. An RTD is the most accurate temperature sensor.

2) Soil Moisture Sensor: Soil Moisture Sensor readings will be given as input to the microcontroller. A customized soil moisture content threshold is set. When the reading is below the threshold, then the microcontroller will change the angle of servo motor to turn ON the Water Pump.

Range : 0 to $45 \%$ volumetric water content in soil (capable of 0 to $100 \%$ VWC with alternate calibration).

The Soil Moisture Sensor uses capacitance to measure dielectric permittivity of the surrounding medium. In soil, dielectric permittivity is a function of the water content. The sensor creates a voltage proportional to the dielectric permittivity, and therefore the water content of the soil.

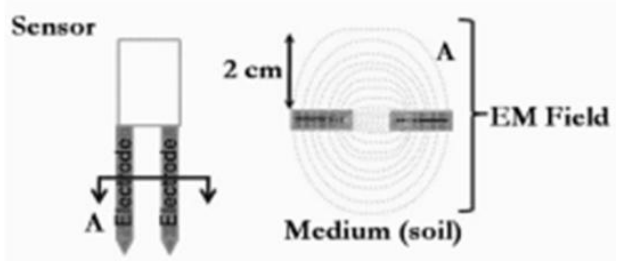

The sensor averages the water content over the entire length of the sensor. There is a 2 $\mathrm{cm}$ zone of influence with respect to the flat surface of the sensor, but it has little or no sensitivity at the extreme edges. The figure above shows the electromagnetic field lines along a cross-section of the sensor, illustrating the $2 \mathrm{~cm}$ zone of influence.

3) Light Dependent Resistor: Light Dependent Resistor readings will be continuously given as input to the microcontroller. Some crops require specific amount of light. If the specific amount of sunlight is not provided to the crops, proper growth of crops is affected. Whenever the intensity of light reduces, the microcontroller will turn on the Light Bulb to maintain the light intensity.

The snake like track shown below is the Cadmium Sulphide (CdS) film which also passes through the sides. On the top and bottom are metal films which are connected to the terminal leads. It is designed in such a way as to provide maximum possible contact area with the two metal films. The structure is housed in a clear plastic or resin case, to provide free access to external light. As explained above, the main component for the construction of LDR is cadmium sulphide (CdS), which is used as the photoconductor and contains no or very few electrons when not illuminated. In the absence of light it is designed to have a high resistance in the range of mega ohms. As soon as light falls on the sensor, the electrons are liberated and the conductivity of the material increases. When the light intensity exceeds a certain frequency, the photons absorbed by the semiconductor give band electrons the energy required to jump into the conduction band. 
This causes the free electrons or holes to conduct electricity and thus dropping the resistance dramatically $(<1$ Kilo ohm).

4) The fourth application of this project is related to sending this data to the mobile device with the help of GSM module. The first application is about maintaining the temperature of that place with the help of temperature sensor (LM-35) and Fan (Motor).

A GSM Module is basically a GSM Modem (like SIM 900) connected to a PCB with different types of output taken from the board - say TTL Output (for Arduino, 8051 and other microcontrollers) and RS232 Output to interface directly with a PC (personal computer). The board will also have pins or provisions to attach mic and speaker, to take out

$+5 \mathrm{~V}$ or other values of power and ground connections. These type of provisions vary with different modules.

\section{CIRCUIT DIAGRAM}

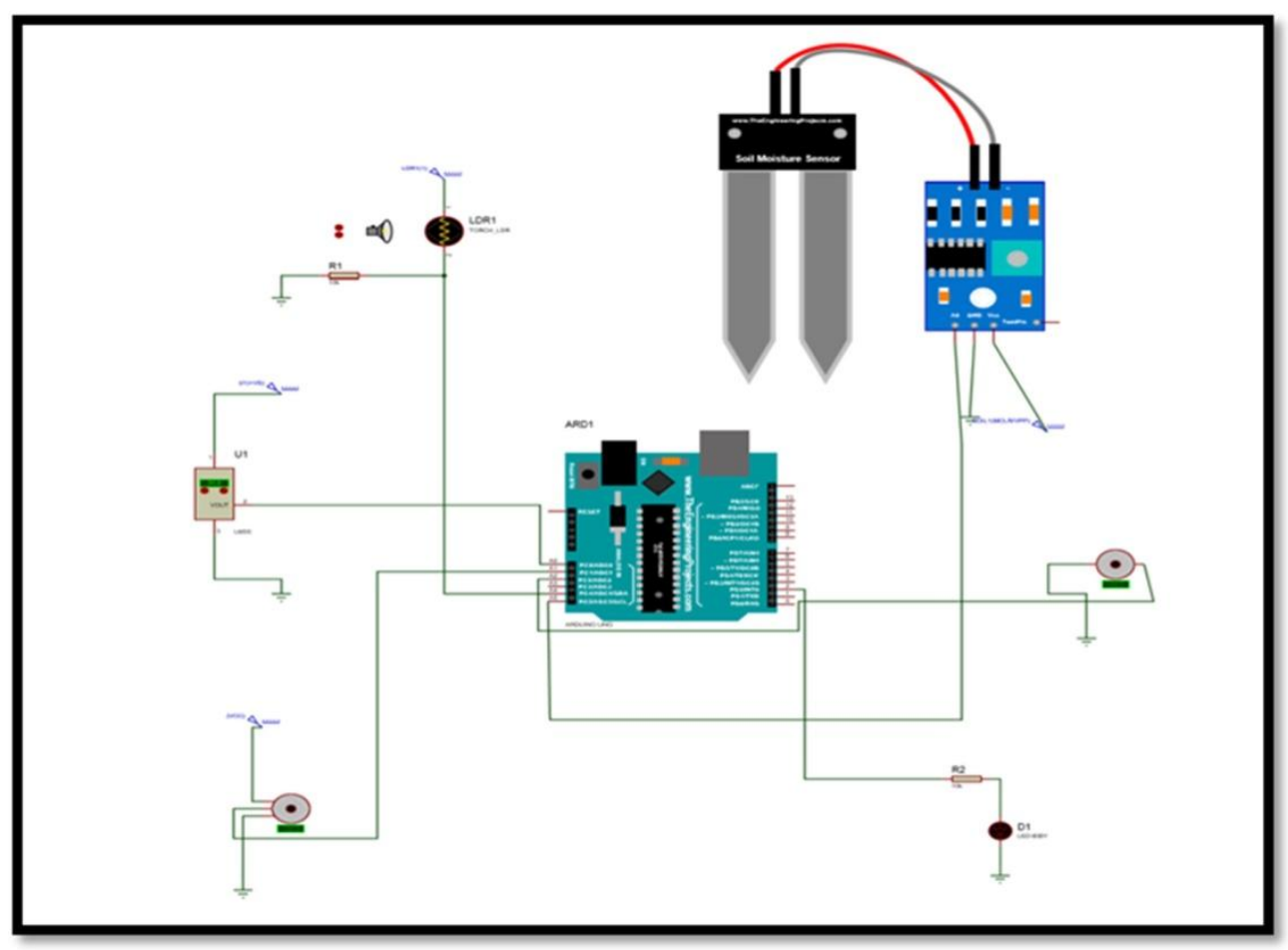




\section{REFERENCES}

[1]. https://scienceprog.com/understanding-and-interfacing-ldr-light-dependent-

$\underline{\text { resistors/ }}$

[2]. https://www.circuitstoday.com/ldr-light-dependent-resistors

[3].https://users.encs.concordia.ca/ bwgordon/arduino_lab3.html

[4]. https://create.arduino.cc/projecthub/electronicprojects/smart-irrigationsystem-using- arduino-uno-afcb31

[5]. https://www.circuitstoday.com/interface-gsm-module-with-arduino

[6]. https://www.electronicsforu.com/resources/gsm-

module\#: :text=A\%20GSM\%20module\%20or\%20a,a\%20GSM $\% 20$ or $\% 20 \mathrm{GPRS} \% 2$

0syste $\mathrm{m}$.

[7]. https://components101.com/lm35-temperature-

sensor\#: :text=LM35\%20is\%20a\%20precession\%20Integrated,C\%20to\%20150\%

$\mathrm{C} 2 \% \mathrm{~B} 0$

C.\&text=There $\% 20$ will\%20be $\% 20$ rise $\% 20$ of,degree $\% 20$ Celsius $\% 20$ rise $\% 20$ in $\% 20$ tem pera ture.

[8]. https://components 101.com/microcontrollers/arduino-uno

[9]. https://www.arduino.cc/en/Main/software

[10].https://en.wikipedia.org/wiki/Soil_moisture_sensor 